Proceedings

\title{
Detection of $A \beta(1-40)$ Protein in Human Serum as a Causative Agent of Alzheimer's Disease by Strain Gauge Cantilever Biosensor Immobilizing Liposome Incorporating Cholesterol ${ }^{+}$
}

\author{
Tomoya Taniguchi ${ }^{1}$, Yuki Murakami ${ }^{1}$, Masayuki Sohgawa ${ }^{2}$, Kaoru Yamashita ${ }^{1}$ \\ and Minoru Noda ${ }^{1, *}$ \\ 1 Kyoto Institute of Technology, Kyoto, Japan; m6621026@edu.kit.ac.jp (T.T.); \\ m5621038@edu.kit.ac.jp (Y.M.); yamashita.kaoru@kit.ac.jp (K.Y.) \\ 2 Niigata University, Niigata, Japan; sohgawa@eng.niigata-u.ac.jp \\ * Correspondence: noda@kit.ac.jp; Tel.: +81-75-724-7443 \\ + Presented at the Eurosensors 2017 Conference, Paris, France, 3-6 September 2017. \\ Published: 7 September 2017
}

\begin{abstract}
We have successfully measured amyloid beta $(A \beta)(1-40)$ protein added in human serum by a $\mathrm{NiCr}$ strain gauge cantilever biosensor immobilized with liposomes incorporating cholesterol. Importantly, we investigated the effect of incorporation of cholesterol in the liposome in order to suppress the interaction between the liposome and many different proteins included in human serum. It was revealed that incorporating cholesterol suppresses the interaction between the proteins other than $A \beta$ in human serum and the liposome. Finally, we detected $A \beta(1-40)$ in human serum with typical chronological behaviors due to $A \beta$ aggregation and fibrillization. Furthermore, as a digital low-pass filtering procedure could reduce external noises, the cantilever sensor immobilized with liposome incorporating cholesterol can detect low-concentrated $\mathrm{A} \beta$ in human serum.
\end{abstract}

Keywords: cantilever; $\mathrm{NiCr}$ strain gauge; biosensor; liposome; amyloid beta; aggregation; fibrillization; interaction; human serum; digital low-pass filtering procedure

\section{Introduction}

Alzheimer's disease (AD) is one of dementia and the mechanism is not clear yet. However amyloid beta $(A \beta)$ is the predominant agent related to $A D$. Also it is recognized that $A D$ is caused by interaction between $A \beta$ and cerebral nerve cells and accumulation of $A \beta$ on them [1,2]. We have reported that our $\mathrm{NiCr}$ strain gauge cantilever microsensor immobilized with liposome as sensing biomolecule could detect $50 \mathrm{nM} \mathrm{A} \beta(1-40)$ [3], which is less than the reported value in AD patients [4]. Though our sensor can detect $50 \mathrm{nM} \mathrm{A} \beta(1-40)$, it's not enough because we are aiming to apply the sensor to diagnose mild cognitive impairment (MCI) patients. Thus it is essential to improve the sensitivity because $A \beta$ concentration might be less than $1 \mathrm{nM}$ in their plasma. Moreover we have to detect the $A \beta$ in human serum of MCI patients. There are a lot of proteins in human serum except $\mathrm{A} \beta$. Therefore it is important for the liposomes to have the high selectivity against $A \beta$. In this work, we tried to measure the $A \beta$ in the human serum including many different proteins that would exhibit interactions with the phospholipids of the liposome.

\section{Experimental Methods}

Figure 1 shows the cantilever microsensor with the human serum added with $A \beta(1-40)$ in a droplet-sealing structure. This cantilever biosensor can sense $A \beta(1-40)$ by interaction between liposomes and $A \beta(1-40)$. Especially we think the sensor can detect oligomer and protofibril as $A \beta$ 
aggregation-intermediates that are more poisonous than $\mathrm{A} \beta$ fibril [3]. This time, we employed 1,2-Ditetradecanoyl-sn-glycero-3-phosphocholine (DMPC) and that incorporated with cholesterol. DMPC liposomes were immobilized on the cantilever by Self-Assembled Monolayer (SAM). Non-aggregated $A \beta$ was dissolved in $0.1 \%$ ammonia water and added with human serum. Non-aggregated $A \beta$ solution was introduced to polydimethylsiloxane (PDMS) droplet-sealing structure. It is possible to measure $A \beta(1-40)$ in target solution for more than $24 \mathrm{hrs}$. We investigated the dynamic behavior between DMPC liposomes and proteins other than $A \beta$ in human serum. In addition, in order to measure less than $1 \mathrm{nM} \mathrm{A} \beta$, we needed to eliminate noise due to temperature fluctuation and the other external one. Therefore we attempted to apply a digital low-pass filtering procedure to improve the sensitivity of this biosensor.

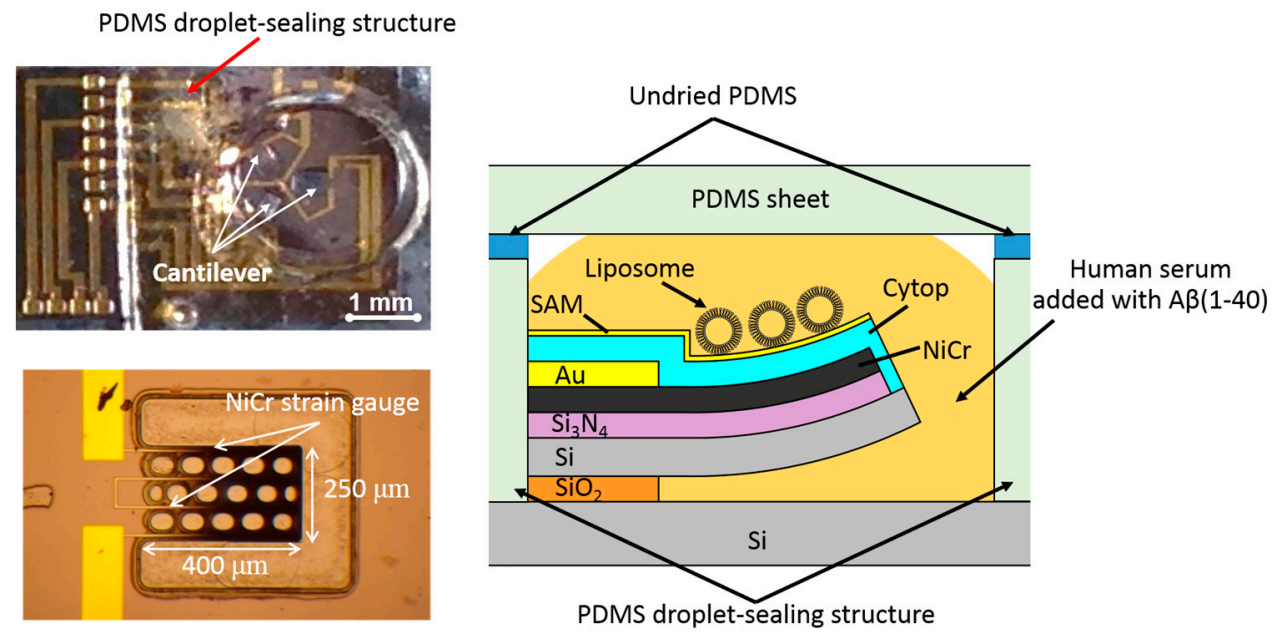

Figure 1. (a) Top surface; (b) a part of cantilever (c) a cross sectional illustration of the cantilever biosensor with PDMS droplet sealing structure in human serum added with A $\beta(1-40)$.

\section{Results and Discussion}

Firstly, we applied a digital low-pass filtering procedure to eliminate noises. Figure 2 shows resistance change rate using 1,2-Dipalmitoyl-sn-glycero-3-phosphorycholine (DPPC) incorporated with cholesterol after adding $50 \mathrm{nM} \mathrm{A} \beta(1-40)$ solution. As shown in Figure 2, the external noise and due to the temperature fluctuation are successfully eliminated by a digital low-pass filtering procedure.

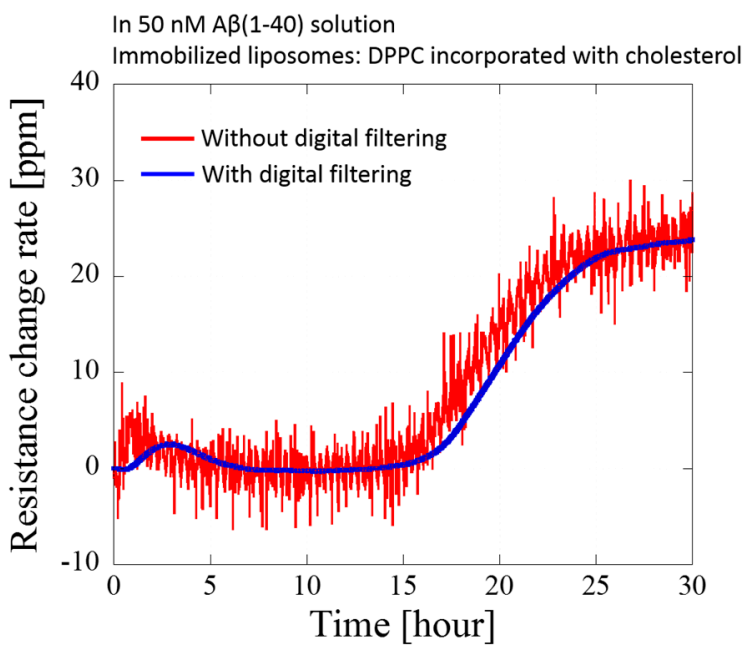

Figure 2. Resistance change rate of microcantilever sensor immobilized with DPPC liposomes incorporated with cholesterol in $50 \mathrm{nM} \mathrm{A} \beta(1-40)$ solution with and without digital filtering procedure. 
Secondly, we investigated interaction between proteins in human serum and liposomes of DMPC and DMPC incorporating cholesterol. As shown in Figure 3, resistance change rate of the cantilever immobilized with DMPC liposomes increased throughout measuring time. On the other hand, resistance of that immobilized with DMPC incorporated with cholesterol was averagely stable. We think that cholesterol can reduce the interaction between the proteins in human serum and DMPC liposomes due to its stabilizing effect on the membrane [5]. This suggests that we can suppress the interaction between the proteins in human serum and liposomes by the incorporation of cholesterol. In other words, the cantilever sensor would be able to detect the dynamic state of $A \beta$ in human serum.

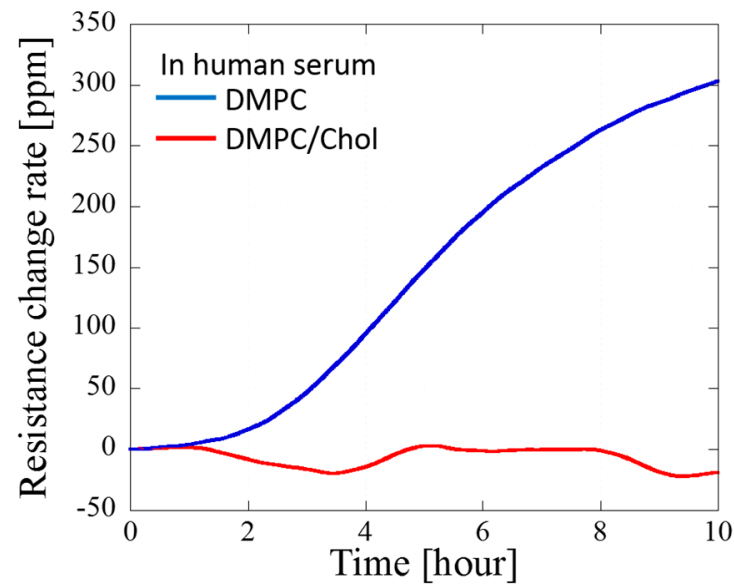

Figure 3. Resistance change rate of the cantilever immobilized with DMPC liposomes and DMPC/Cholesterol liposomes in human serum.

Finally, we tried to detect $1 \mu \mathrm{M} A \beta(1-40)$ dissolved in human serum. The result is shown in Figure 4. From 0 to $11 \mathrm{~h}$, the resistance of the cantilever increased because monomeric $A \beta(1-40)$ interacted DMPC [6] and DMPC did not interact proteins in human serum. After that, until $15 \mathrm{~h}$, the resistance rapidly increased. This indicates that cholesterol enhanced the interaction between DMPC liposomes and $A \beta(1-40)$ [7]. Furthermore, after $15 \mathrm{~h}$, the increasing ratio of the resistance change rate of the cantilever becomes decreased. Although membrane fluidity is increasing by interacting proteins, the decrease suggests that a series of aggregation and fibrillization become matured and saturated. Therefore, we consider that the micro cantilever biosensor can detect the typical chronological behaviors of $A \beta(1-40)$. Consequently, we expect that the cantilever biosensor can detect low $A \beta$ concentration in human serum by utilizing incorporation of cholesterol and the digital filtering procedure.

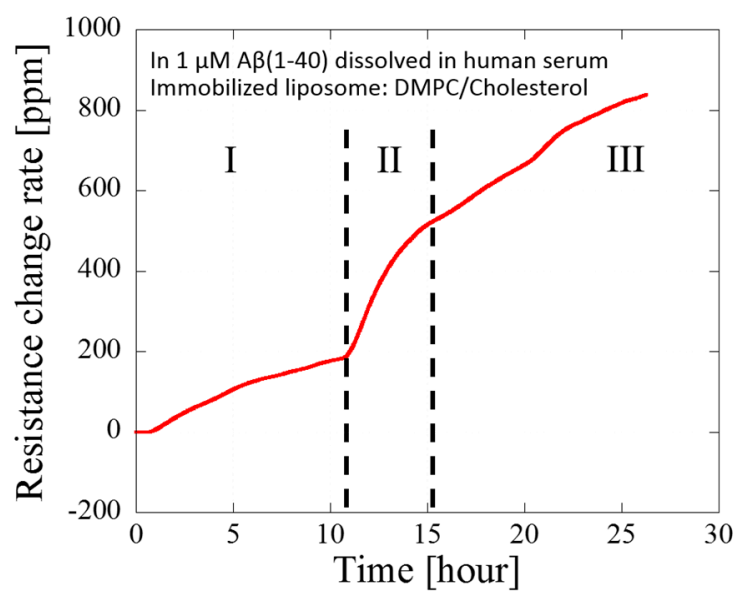

Figure 4. The change in resistance of the cantilever biosensor immobilized with DMPC/Cholesterol in $1 \mu \mathrm{M} A \beta(1-40)$ dissolved in human serum: (I) monomeric $\mathrm{A} \beta(1-40)$, (II) aggregation of $\mathrm{A} \beta(1-40)$ and (III) mature $A \beta(1-40)$ fibril. 


\section{Conclusions}

We attempted to detect $\mathrm{A} \beta(1-40)$ protein added in human serum using a $\mathrm{NiCr}$ strain gauge cantilever biosensor immobilized with liposomes incorporating cholesterol so as to apply the sensor for a diagnosis of $\mathrm{AD}$ and especially $\mathrm{MCI}$ patients. It is possible to eliminate external noises by a digital filtering procedure. It shows that incorporation of cholesterol can suppress the interaction between DMPC liposomes and proteins in human serum. In addition, we detected the dynamic behavior of $A \beta(1-40)$ through aggregation and fibrillization in human serum by the sensor immobilized DMPC liposomes incorporated with cholesterol. We conclude that the cantilever biosensor immobilized with liposomes incorporating cholesterol can detect low-concentrated $A \beta$ in human serum.

Acknowledgments: This research was supported in part by a Grant-in-Aid for Scientific Research (KAKENHI Grant No. 25249048 and 26630157) from the Japan Society for the Promotion of Science (JSPS).

Conflicts of Interest: The authors declare no conflict of interest.

\section{References}

1. Nussbaum, R.L.; Ellis, C.E. Alzheimer's disease and Parkinson's disease. N. Engl. J. Med. 2003, 348, 1356-1364.

2. Hardy, J.; Selkoe, D.J. The amyloid hypothesis of Alzheimer's disease: Progress and problems on the road to therapeutics. Science 2003, 297, 353-356.

3. Zhang, Z.; Murakami, Y.; Taniguchi, T.; Sohgawa, M.; Yamashita, K.; Noda, M. A cantilever-based biosensor for real-time monitoring of interactions between amyloid- $\beta(1-40)$ and membranes comprised of phosphatidylcholine lipids with different hydrophobic acyl chains. Electroanalysis 2017, 29, 722-729.

4. Coppus, A.M.W.; Schuur, M.; Vergeer, J.; Janssens, A.C.J.W.; Oostra, B.A.; Verbeek, M.M.; van Duijn, C.M. Plasma $\beta$ amyloid and the risk of Alzheimer's disease in down syndrome. Neurobiol. Aging 2012, 33, 1988-1994.

5. Komura, S.; Andelman, D. Physical aspects of heterogeneities in multi-component lipid membranes. Adv. Coll. Int. Sci. 2014, 208, 34-46.

6. Lockhart, C.; Klimov, D.K. Calcium enhances binding A $\beta$ monomer to DMPC lipid bilayer. Biophys. J. 2015, 108, 1807-1818.

7. Matsuzaki, K. Physicochemical interactions of amyloid $\beta$-peptide with lipid bilayers. Biochim. Biophys. Acta 2007, 1768, 1935-1942.

(C) 2017 by the authors. Licensee MDPI, Basel, Switzerland. This article is an open access article distributed under the terms and conditions of the Creative Commons Attribution (CC BY) license (http://creativecommons.org/licenses/by/4.0/) 